\title{
Performance Evaluation of FSO Link Under NRZ-RZ Line Codes, Different Weather Conditions and Receiver Types in the Presence of Pointing Errors
}

\author{
Nazmi A. Mohammed, Amr S. El-Wakeel* and Mostafa H. Aly \\ Electronics and Communications Engineering Department, Arab Academy for Science, Technology and Maritime \\ Transport, Egypt
}

\begin{abstract}
Performance evaluation for a free space optical (FSO) link with latest wireless optical communications (WOC) vendor's networks specifications is presented. Analysis is performed for non return to zero (NRZ)-return to zero (RZ) line codes with various operating wavelengths using APD and PIN photodiodes receivers. The study includes the effect of atmospheric attenuation due to scattering effects for different weather conditions in the presence of pointing error.

Maximum pointing error, received signal's power and bit error rate (BER) levels are indicators for performance evaluation. NRZ line code with $1550 \mathrm{~nm}$ operating wavelength and an APD receiver shows the best performance for the proposed FSO link. In the presence of moderate fog weather condition, $11.85 \mu \mathrm{rad}$ is the maximum pointing error and - 41.09 $\mathrm{dBm}$ is achieved for NRZ-APD at $1550 \mathrm{~nm}$ in order to maintain $\mathrm{BER}<10^{-9}$.
\end{abstract}

Keywords: Free space optical (FSO) communications, wireless optical communication (WOC), non return to zero (NRZ), return to zero (RZ), avalanche photodiode (APD), PIN photo diode, scattering, bit error rate (BER), pointing error.

\section{INTRODUCTION}

Free Space Optical (FSO) Communication nowadays is one of the major hot topics in the world of optical and wireless communications. This type of cable-less optical communications technology uses a highly directed narrow light beam to transmit data between two fixed points [1]. It can avoid some challenges facing optical fiber communications such as high cost of digging roads, impractical physical connection between transmitters and receivers. Also, it can be presented to be an alternative or an upgrade for long distance wireless communications systems (up to few kilometers) [2]. Some advantages are: 1) No need for licensed frequency band allocation. 2) Easy to install. 3) Absence of radiation hazards of radio frequency. 4) Immunity to interference. 5) High data rates $[1,3]$.

Achieving acceptable performance for a practical FSO link requires to overcome some major challenges, at the transmitter, determination of modulation techniques $[4,5]$, suitable light sources [3] and transmitting wavelengths [6, 7]. Also, estimating transmitting power levels and pointing errors are various obstacles facing transmitter design [7-9].

There are several challenges facing the channel performance. First, free space path loss phenomenon $[3,6,10]$. Second, investigating effect of different weather conditions

*Address correspondence to this author at the Electronics and Communications Engineering Department, Arab Academy for Science, Technology and Maritime Transport, Egypt; Tel: (+202) 22685615-6; Fax: (+202) 22685892;

E-mail: amr.s.elwakeel@gmail.com that appears in the studies dealing with scattering [11-13], turbulence [14] and scintillation [12, 15].

FSO link receiver strongly affects the behavior of the link. Types of detectors $[3,14,16]$, various sources of noise $[6,17]$ and error correction techniques for maintaining desired bit error rates accepted levels are the top factors that should be considered in the design of practical FSO receivers $[4,8,18]$. For FSO links transmitters, many modulation techniques are used such as NRZ, RZ [4, 19], PPM [16], BPSK [19] and DQPSK [4]. Different types of light sources are used in FSO like LED [3], VSCEL lasers [1], QCL [6, 7]. Various wave lengths are evaluated such, $785 \mathrm{~nm}[11], 830$ $\mathrm{nm}$ [19], $850 \mathrm{~nm}$ [12], $950 \mathrm{~nm}[12], 1550 \mathrm{~nm}[11,6]$ and $10,000 \mathrm{~nm}[6]$. Pointing errors loss factors are discussed [6, $7,8,9]$. For FSO link channel, effect of scattering is evaluated through Kim's model [11-13], Kruse's Model [12, 13], Al Naboulsi's advection fog model [13] and Al Naboulsi's convection (radiation) fog model [13], phenomena of turbulence and scintillation [15] are evaluated in the log normal channel model [16], negative exponential channel model [16] and gamma-gamma model [14]. APD and PIN are introduced as FSO link receivers $[3,14,16]$, where its performance is affected by thermal noise $[6,17]$, shot noises $[6,17]$ and sometimes FSO detector noise is estimated by AWGN [14]. Error correction techniques are used to maintain desired BER levels such as LDPC codes $[8,18]$ and convolutional codes [20].

In this paper, we have embarked an FSO link performance evaluation under wide range of commercial design parameters. In our evaluation, an FSO link (Transmitter- 
Channel-Receiver) is tested under: 1) Most famous (for FSO) modulation techniques, 2) Practical transmitter wavelengths, 3) Different levels of various weather conditions, 4) FSO receivers including physical specifications. Performance evaluation for the designed link is determined by estimation of the maximum pointing error (in transmitter and receiver) required to achieve $\mathrm{BER}<10^{-9}$ (desired value for all practical FSO links [20]).

The remainder of this paper is organized as follows. The mathematical review is presented in Section 2. Based on the theory presented, a numerical analysis and system simulation are carried out to evaluate the performance of the proposed FSO link in Section 3. This is followed by conclusion in Section 4.

\section{MATHEMATICAL MODEL}

\subsection{Atmospheric Attenuation}

One of the challenges of the FSO channel which may lead to signal loss and link failure is the atmospheric attenuation [1]. Scattering and turbulence phenomena highly affect the power of the transmitted signal $[11,15]$. Rayleigh scattering, Mie scattering and geometrical scattering are the types of scattering that are related to the size of particles in the atmosphere and the wavelength of the transmitted signal of an FSO link [11, 12].

Atmospheric attenuation due to scattering is modeled for different weather conditions and particles size. Kim [11,12], Kruse $[12,13]$ and Al-Naboulsi $[13,21]$ are some of the famous models that present the effect of scattering on the transmitted beam in FSO channel. The Beers-Lambert law represents the relation between the power of the transmitted signal and the received signal in the presence of atmospheric attenuation [11].

If $\boldsymbol{P}_{\boldsymbol{T}}$ and $\boldsymbol{P}_{\boldsymbol{R}}$ are the transmitted and the received power and $\boldsymbol{\alpha}$ is atmospheric attenuation coefficient and $\boldsymbol{Z}$ is the link range, then

$P_{R}=P_{T} \exp (-\alpha Z)$

The coefficient of atmospheric attenuation depends on the type of scattering, signal wavelength, size of the particles of the atmosphere and the link visibility [11]. In the link, we introduce the atmospheric attenuation coefficient as calculated through Kim's Model [11, 12]

$\alpha=3.91 / V(\lambda / 550 \mathrm{~nm})^{-q}$

$\boldsymbol{V}$ is the visibility and $\boldsymbol{q}$ is the size distribution of the scattering particles. The attenuation of the transmitted signal can be estimated from the previous model to various weather conditions using

$$
\begin{aligned}
q & =1.6 \text { for visibility }(V>50 \mathrm{~km}) \\
& =1.3 \text { for visibility }(6 \mathrm{~km}<V<50 \mathrm{~km}) \\
& =0.16 V+0.34 \text { for visibility }(1 \mathrm{~km}<V<6 \mathrm{~km}) \\
& =V-0.5 \text { for visibility }(0.5 \mathrm{~km}<V<1 \mathrm{~km}) \\
& =0 \text { for visibility }(V<0.5 \mathrm{~km})
\end{aligned}
$$

\subsection{Link Budget}

Atmospheric attenuation, free space path loss, transmitter and receiver gain, types of detectors, efficiencies and pointing loss factors are considered the main factors that highly affect link budget calculations. Friis transmission formula is introducing the link budget model $[6,7,10]$

$P_{R}=P_{T} \eta_{T} \eta_{R}(\lambda / 4 \pi Z)^{2} G_{T} G_{R} L_{T} L_{R} \exp (-\alpha Z)$

The free space path loss $[6,7]$ is represented by the factor $(\lambda / 4 \pi z)^{2}$.

where $\lambda$ is the signal wavelength, $\boldsymbol{\eta}_{T}$ and $\boldsymbol{\eta}_{R}$ are transmitter and receiver efficiencies, $\boldsymbol{G}_{\boldsymbol{T}}$ and $\boldsymbol{G}_{\boldsymbol{R}}$ are the transmitter and receiver gain. When the transmitter is assumed to be uniformly illuminated from a circle aperture, the out beam cross section is considered as a Gaussian beam and the receiver antenna is a circular aperture $[6,7]$. The transmitter and receiver gain expressions are given by

$G_{T}=\left(\pi D_{T} / \lambda\right)^{2}$

$G_{R}=\left(\pi D_{R} / \lambda\right)^{2}$

Where $\boldsymbol{D}_{T}$ and $\boldsymbol{D}_{R}$ are the transmitter and receiver aperture diameters.

Assuming Gaussian beam, the transmitter and receiver pointing loss factors, respectively, are $\boldsymbol{L}_{\boldsymbol{T}}$ and $\boldsymbol{L}_{\boldsymbol{R}}$

$L_{T}=\exp \left(-G_{T}\left(\theta_{T}\right)^{2}\right)$

$L_{R}=\exp \left(-G_{R}\left(\theta_{R}\right)^{2}\right)$

Where $\boldsymbol{\theta}_{\boldsymbol{T}}$ and $\boldsymbol{\theta}_{\boldsymbol{R}}$ are transmitter's and receiver's pointing errors.

\subsection{Noise Model and BER Evaluation}

Optical signal's power at the receivers end $\boldsymbol{P}_{\boldsymbol{R}}$ is converted into electrical current through the photodetectors (APDPIN) [22].

Thermal noise, shot noise and back ground noises are the main noise mechanisms lead to current fluctuations and non precise bit detection $[6,7,22]$. Only effect of the thermal noise is considered in the proposed link analysis. The total current $\boldsymbol{I}$ consists of two components $\boldsymbol{I}_{p}$ and $\boldsymbol{i}_{T}$.

$I=I_{P}+i_{T}$

$\boldsymbol{I}_{\boldsymbol{P}}$ is the average current without noise effect. $\boldsymbol{R}$ and $\boldsymbol{P}_{\boldsymbol{R}}$ are responsivity and received power respectively.

$I_{n}=R P_{R}$

$\boldsymbol{i}_{T}$ is the thermal noise current fluctuation that is modeled as a stationary Gaussian random process with variance $\sigma_{T}{ }^{2}$

$\sigma_{T}^{2}=\left(4 K T / R_{L}\right) \Delta f$

$\boldsymbol{K}$ is Boltzmann's constant, $\boldsymbol{T}$ is absolute temperature, $\boldsymbol{R}_{\boldsymbol{L}}$ is the receiver's load resistance and $\Delta \boldsymbol{f}$ is the effective noise bandwidth. The total variance of current fluctuations $\sigma$ as following

$\Delta I=I-I_{P}$

$\sigma^{2}=\sigma_{T}^{2}$ 
Table 1. FSO Link Parameters

\begin{tabular}{|c|c|c|c|}
\hline Parameter & \multicolumn{2}{|c|}{ Symbol } & Value \\
\hline Transmission Rate & \multicolumn{2}{|c|}{ Bit rate } & $1.25 \mathrm{Gbps}$ \\
\hline Link Distance & \multicolumn{2}{|c|}{$Z$} & $1 \mathrm{~km}$ \\
\hline \multirow{2}{*}{$\begin{array}{l}\text { Optical Transmitted Power } \\
\text { (Gaussian Beam) }\end{array}$} & \multirow{2}{*}{$P_{T}$} & NRZ & $22.6 \mathrm{dBm}$ \\
\hline & & $\mathrm{RZ}$ & $20.7 \mathrm{dBm}$ \\
\hline Transmitter and Receiver Apertures & \multicolumn{2}{|c|}{$D_{T}, D_{R}$} & $10 \mathrm{~cm}$ \\
\hline Transmitter and Receiver Optics Efficiency & \multicolumn{2}{|c|}{$\eta T \quad \eta R$} & $0.75-0.8$ \\
\hline APD Responsivity & \multicolumn{2}{|c|}{$R$} & $0.8-52 \mathrm{~A} / \mathrm{w}$ \\
\hline PIN Responsivity & \multicolumn{2}{|c|}{$R$} & $0.55-0.85 \mathrm{~A} / \mathrm{w}$ \\
\hline Load Resistance & \multicolumn{2}{|c|}{$R_{L}$} & $100 \Omega$ \\
\hline Low Pass Filter Cutoff Frequency (at receiver) & \multicolumn{2}{|c|}{-} & $0.75^{*}$ Bit rate \\
\hline
\end{tabular}

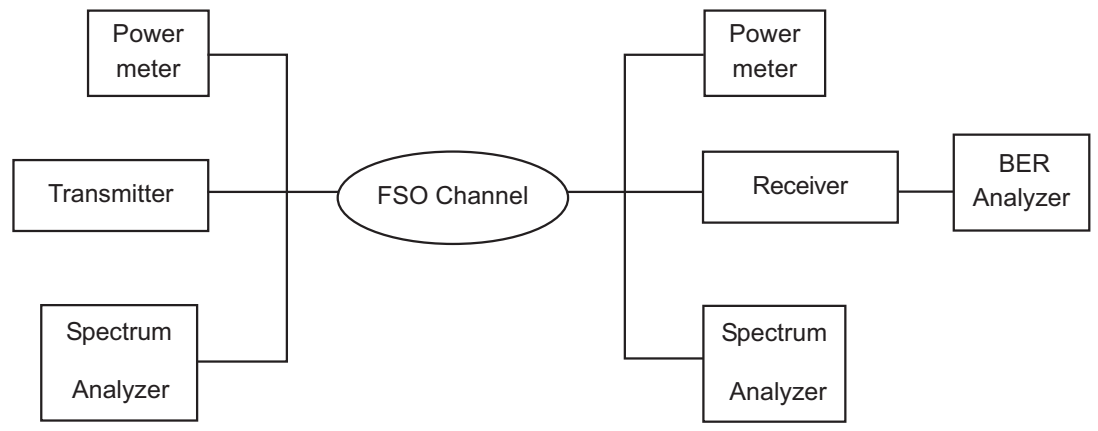

Fig. (1). FSO link structure.

BER is a criterion to evaluate a performance of a digital system, where it is the probability of non precision decision for the received bit stream. As $P(0 / 1)$ is the probability of deciding 0 when 1 is received and $P(0 / 1)$ is the probability of deciding 1 when 0 is received.

$B E R=\frac{1}{2}[P(0 / 1)+P(1 / 0)]$

The BER expression for APD and PIN is derived as following [27]:

$$
B E R=\frac{1}{2} \operatorname{erfc}\left(\frac{Q}{\sqrt{2}}\right) \approx \frac{\exp \left(-Q^{2} / 2\right)}{Q \sqrt{2 \pi}} \text { For } \boldsymbol{Q}>3
$$

$Q=\frac{I_{1}-l_{0}}{\sigma_{1}+\sigma_{0}}$

$\boldsymbol{I}_{\boldsymbol{1}}$ and $\boldsymbol{I}_{\boldsymbol{o}}$ are the average current for 1 and 0 bits respectively where $\sigma_{1}{ }^{2}$ and $\sigma_{o}{ }^{2}$ are the variances with equal value.

\subsection{FSO Link Specifications}

In this section, we introduce in Table $\mathbf{1}$ important FSO parameters that formulate the designed link and kept constant during all the following analysis. These values are chosen to meet the latest practical FSO links and are provided by various FSO vendors [23-25]. Other various specs and parameters mentioned in Section 1 are chosen from FSO vendors to support following performance analysis [26, 27].
For the transmitter a pseudo random bit generator is used to generate a random bit stream to be transmitted then a NRZRZ pulse generators are used as signal modulators, Direct Modulated laser is used to transmit the message bit stream. Chosen transmission power for NRZ-RZ mentioned in Table $\mathbf{1}$ are in the practical range of FSO vendors [23-25]. The difference between NRZ and RZ transmitted power is due to that the intensity of the output of a laser varied according to the modulating data format and the RZ power is less than NRZ power as known in digital communication [29,30]. NRZ-RZ Gaussian BER technique is used in BER estimation in the link's analysis [22]. The receiver threshold power and sensitivity are in the range of $-30 \mathrm{dBm}$ for PIN to $-40 \mathrm{dBm}$ for APD $[18,22,28]$. Different types of noise affect the BER levels of the FSO link like thermal and shot noise. In this work, only thermal noise is considered [6, 7, and 22].

\section{RESULTS AND DISCUSSIONS}

\subsection{Introduction}

The FSO communications proposed link is illustrated in Fig. (1). Link performance is evaluated for modulation techniques and practical operating wavelengths; this is modeled in the transmitter block diagram. The designed link is evaluated under different weather conditions in the presence of free space path loss. Finally, APD and PIN photodetectors are used to test the performance of the proposed link. Optical spectrum analyzers, optical power meters and BER analyzer 


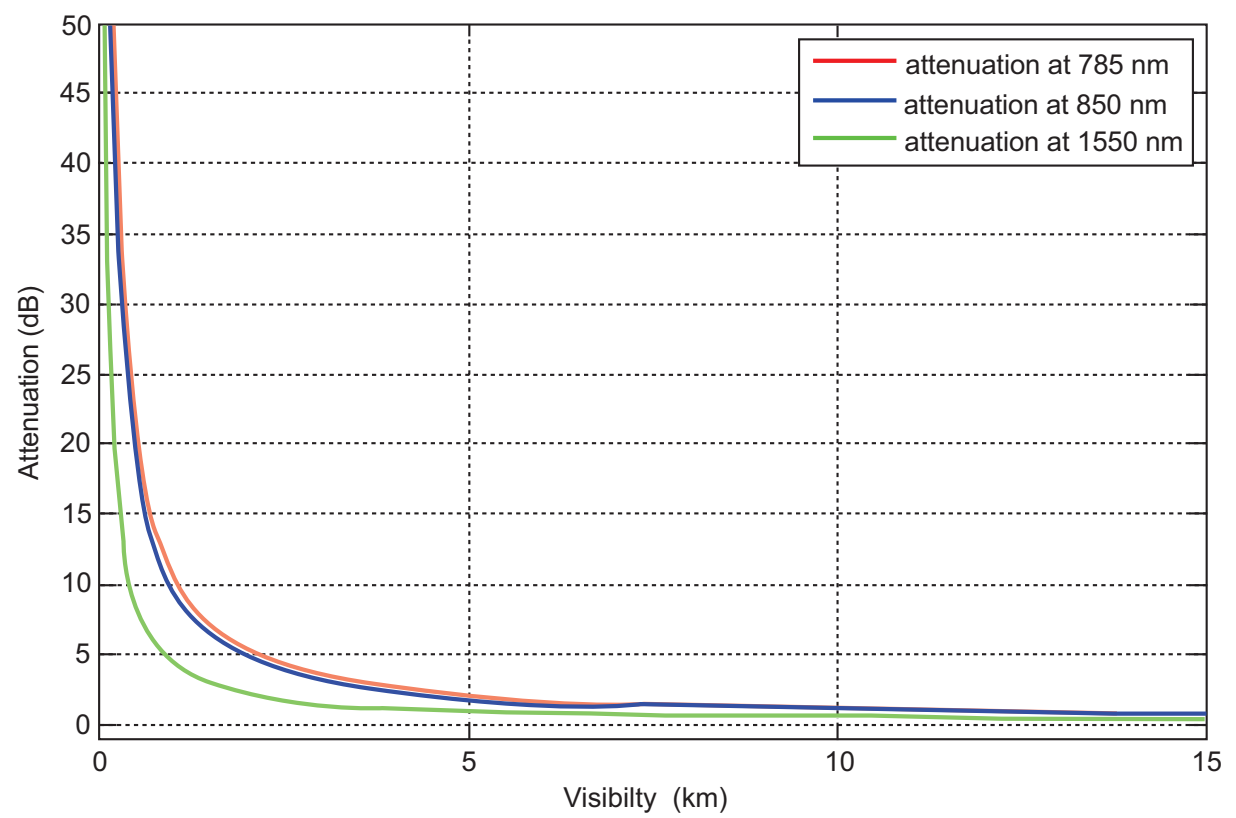

Fig. (2). Atmospheric attenuation Vs visibilty at different weather conditions for various wavelengths.

Table 2. Atmospheric Attenuation at Different Weather Conditions for Various Wavelengths

\begin{tabular}{|c|c|c|c|c|}
\hline Weather Condition & Visibility (km) & $\begin{array}{c}\lambda=\mathbf{7 8 5} \mathbf{~ n m} \\
\text { Attenuation(dB/km) }\end{array}$ & $\begin{array}{c}\lambda=\mathbf{8 5 0} \mathbf{n m} \\
\text { Attenuation(dB/km) }\end{array}$ & $\begin{array}{c}\lambda=\mathbf{1 5 5 0} \mathbf{~ n m} \\
\text { Attenuation(dB/km) }\end{array}$ \\
\hline \hline Clear air & 23 & 0.5 & 0.4 & 0.1 \\
\hline Haze & 2 & 6.7 & 6.4 & 4.2 \\
\hline Light fog & 0.8 & 19.1 & 18.6 & 15.5 \\
\hline Moderate fog & 0.6 & 27.3 & 27 & 25.5 \\
\hline
\end{tabular}

are used to determine the transmitted and received signal power levels and the system BER. Optisystem 10.0 from optiwave Inc. is used to construct the presented FSO link and for the link analysis, Matlab 2008 is used in the atmospheric loss factors calculations and the loss factors is fed into the constructed link in the Optisystem.

The obtained results will be discussed as follows: the effect of various atmospheric conditions on signal power at different wavelengths is presented in Sec. 3.2. The performance of the FSO link with different wavelengths and link specs is discussed in Sections 3.3, 3.4 and 3.5. In Sec. 3.6, a comprehensive performance evaluation for the proposed FSO system is presented.

\subsection{Effect of Atmospheric Conditions}

Atmospheric weather conditions have a notable effect on the performance of FSO links. Effect of different weather conditions is related to the size distribution of the scattering particles $\boldsymbol{q}$ and the visibility $\boldsymbol{V}$. Effect on signal power levels due to previous dependence and operating wavelengths is shown in Fig. (2).

For clear air and high visibility $(\boldsymbol{V}=23 \mathrm{~km})$, the effect of atmosphere on the signal power levels is almost negligible for all studied wavelengths. The situation changes at haze and fog conditions. For haze $(\boldsymbol{V}=2 \mathrm{~km})$, the visibility starts to decrease and the effect of the scattering particles appears.
As shown in Fig. (2), the effect of haze on the signal power varies with different wavelengths where the largest attenuation is observed for $785 \mathrm{~nm}$. The results obtained applies kim's model presented in section 2.1 [11].

At light $(\boldsymbol{V}=0.8 \mathrm{~km})$ and moderate fog $(\boldsymbol{V}=0.6 \mathrm{~km})$, $1550 \mathrm{~nm}$ achieves least attenuation levels. At low visibilities, where heavy fog $(\boldsymbol{V}<0.5 \mathrm{~km})$, the attenuation levels increase rapidly on all the various wavelengths $[11,12]$. Table 2 summarizes the results obtained from Fig. (2) and will be used in all following link analysis.

\subsection{Performance Analysis for FSO Link at $785 \mathrm{~nm}$}

Performance evaluation of the proposed link at $785 \mathrm{~nm}$ with NRZ-RZ line codes and APD-PIN receivers under various weather conditions is analyzed in this section. Table 3 shows the maximum pointing error evaluated for the designed link to target a BER $<10^{-9}$. In this section and following sections, to provide a clear vision on the system performance of FSO link, a specific weather condition with an operating wavelength will be analyzed under proposed types of modulation techniques and receiver types. In this section, clear air with $785 \mathrm{~nm}$ will be studied.

For clear air with APD receiver, the maximum pointing error for NRZ-APD is $7.55 \mu \mathrm{rad}$, same as RZ-APD. Pervious identical pointing error level is almost common (in behavior) for all weather conditions but the case of RZ-APD signal is 
Table 3. Maximum Pointing Errors and Received Signal Power at Different Weather Conditions for NRZ-RZ Line Codes and APDPIN Receivers, $\lambda=785 \mathrm{~nm}$

\begin{tabular}{|c|c|c|c|c|c|c|c|c|}
\hline \multirow{3}{*}{$\begin{array}{c}\text { Modulation Technique } \\
\text { Receiver Type }\end{array}$} & \multicolumn{4}{|c|}{ NRZ } & \multicolumn{4}{|c|}{$\mathbf{R Z}$} \\
\hline & \multicolumn{2}{|c|}{ APD } & \multicolumn{2}{|c|}{ PIN } & \multicolumn{2}{|c|}{ APD } & \multicolumn{2}{|c|}{ PIN } \\
\hline & $\begin{array}{c}\text { Max. pointing } \\
\text { error } \\
(\mu \mathrm{rad})\end{array}$ & $\begin{array}{l}\text { Received } \\
\text { power } \\
(\mathrm{dBm})\end{array}$ & $\begin{array}{c}\text { Max. pointing } \\
\text { error } \\
(\mu \mathrm{rad})\end{array}$ & $\begin{array}{l}\text { Received } \\
\text { power } \\
(\mathrm{dBm})\end{array}$ & $\begin{array}{l}\text { Max. pointing } \\
\text { error } \\
(\mu \mathrm{rad})\end{array}$ & $\begin{array}{l}\text { Received } \\
\text { power } \\
(\mathrm{dBm})\end{array}$ & $\begin{array}{l}\text { Max. pointing } \\
\text { error } \\
(\mu \mathrm{rad})\end{array}$ & $\begin{array}{c}\text { Received } \\
\text { power } \\
(\mathrm{dBm})\end{array}$ \\
\hline Clear air & 7.55 & -39.09 & 7.15 & -31.19 & 7.55 & -41.02 & 7.15 & -33.12 \\
\hline Haze & 7.25 & -39.12 & 6.85 & -31.55 & 7.25 & -41.04 & 6.85 & -33.48 \\
\hline Light fog & 6.65 & -39.91 & 6.15 & -32.29 & 6.6 & -40.92 & 6.15 & -33.22 \\
\hline Moderate fog & 6.15 & -39.21 & 5.65 & -31.28 & 6.15 & -41.14 & 5.65 & -33.21 \\
\hline
\end{tabular}

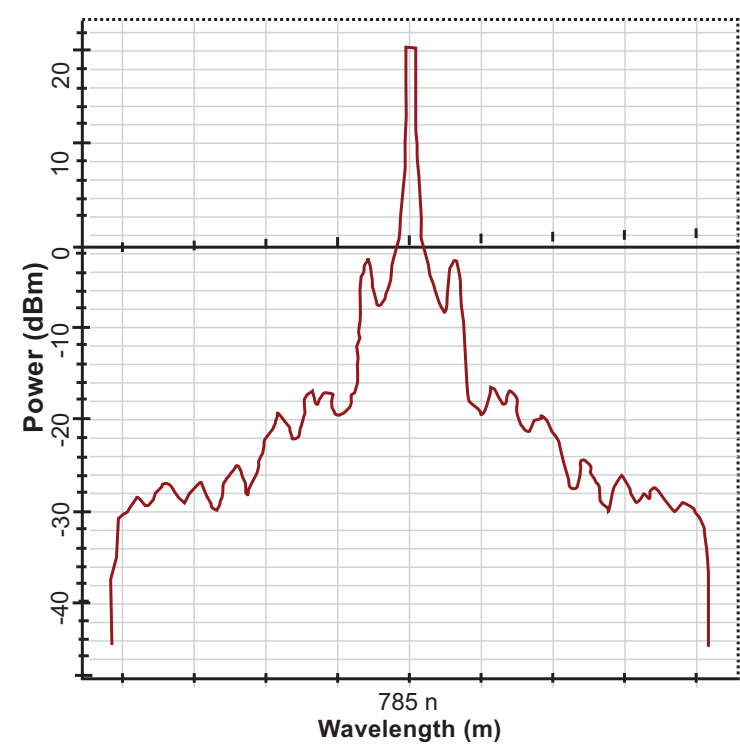

(a)

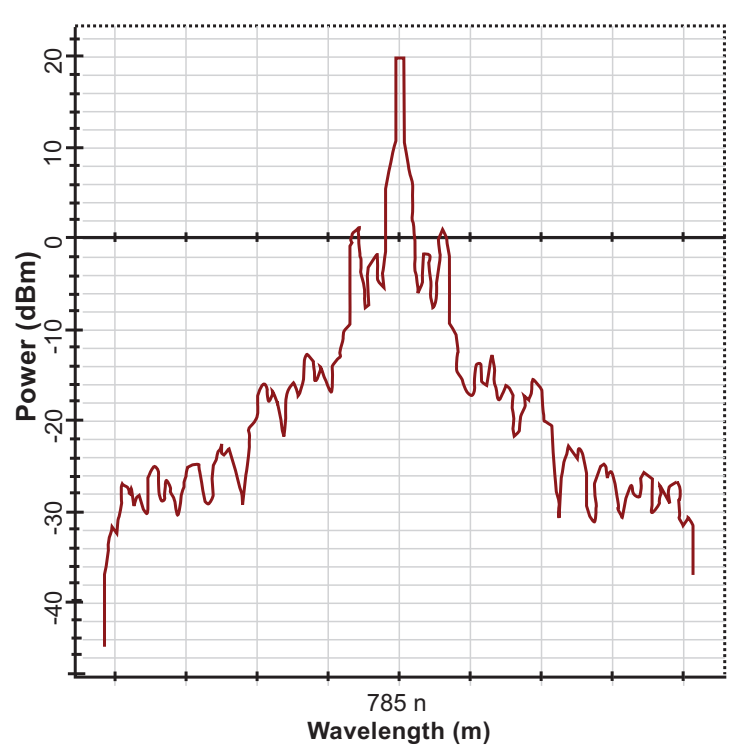

(b)

Fig. (3). Transmitted signal Vs wavelength at $\lambda=785 \mathrm{~nm}$ (a) NRZ (b) RZ.

attenuated more than NRZ-APD. BER analysis for clear air, at the same maximum pointing error, NRZ-APD case achieves BER of $10^{-14}$ and RZ-APD case achieves BER of $10^{-11}$. As a conclusion for any weather condition at $785 \mathrm{~nm}$ with APD receiver, NRZ line code achieves a better performance than RZ by providing higher level of received signal and better BER level.

For clear air with PIN receiver, NRZ-PIN and RZ-PIN have the same maximum pointing error of $7.15 \mu \mathrm{rad}$ (common in behavior to all weather conditions). NRZ-PIN exposed to less attenuation than RZ-PIN while $10^{-13}$ BER is achieved for NRZ-PIN and $10^{-11}$ for RZ-PIN. Similar conclusion (as APD receiver case) can be carried out for any weather conditions at $750 \mathrm{~nm}$ showing that NRZ is better than RZ.

The analysis in this section provides that, APD receiver allows larger pointing error than PIN. This is mainly due to APD higher range of possible received power levels as a result of its physical specifications (dark current, responsivity, gain and thermal noise [26, 27]).
As the weather conditions get worse the loss factor of atmospheric attenuation increases and it is reflected on the maximum allowable pointing error in order to keep the BER lower than $10^{-9}$.

For clear justification, Fig. (3a) and (b) shows input signal at transmitter of the proposed system for NRZ and RZ line codes, respectively, at $785 \mathrm{~nm}$. These transmitted signals are used with the same power level mentioned in Table $\mathbf{1}$ for all the following sections changing only the operating wavelength. The NRZ-APD case presents the best performance where RZ-PIN case presents the worst performance in the $785 \mathrm{~nm}$ link in the presence of clear air. The received signal power Vs wavelength for NRZ-APD and RZ-PIN are shown in Fig. (4a) and (b).

Although NRZ lunched in the atmosphere with higher power than RZ (for the reasons mentioned in section 2.4) that reflected on maximum received power and maximum allowable pointing error. The simulation results shows that the thermal noise in APD and PIN model introduced in section 3.3 apply minor effects on the received power in APD 


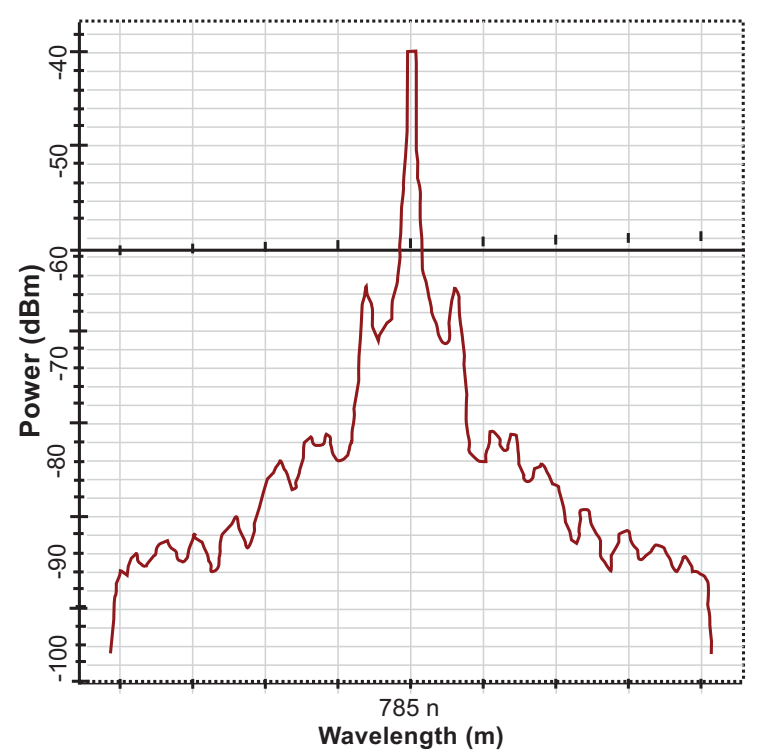

(a)

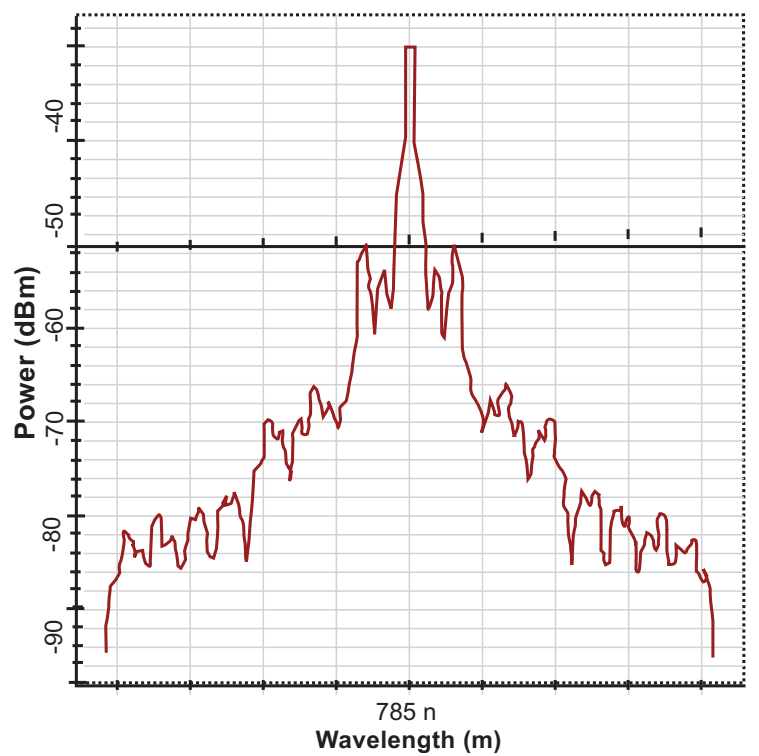

(b)

Fig. (4). Received power Vs wavelength in the presence of clear air at $\lambda=785 \mathrm{~nm}$ (a) NRZ-APD (b) RZ-PIN.

Table 4. Maximum Pointing Errors and Received Signal Power at Different Weather Conditions for NRZ-RZ line Codes and APDPIN Receivers, $\lambda=850 \mathrm{~nm}$

\begin{tabular}{|c|c|c|c|c|c|c|c|c|}
\hline Modulation Technique & \multicolumn{4}{|c|}{ NRZ } & \multicolumn{4}{c|}{ APZ } \\
\hline Receiver Type & \multicolumn{2}{|c|}{ APD } & \multicolumn{2}{c|}{ PIN } & \multicolumn{2}{c|}{ PIN } \\
\hline \hline & $\begin{array}{c}\text { Max. pointing } \\
\text { error } \\
(\mu \mathrm{rad})\end{array}$ & $\begin{array}{c}\text { Received } \\
\text { power } \\
(\mathrm{dBm})\end{array}$ & $\begin{array}{c}\text { Max. pointing } \\
\text { error } \\
(\mu \mathrm{rad})\end{array}$ & $\begin{array}{c}\text { Received } \\
\text { power } \\
(\mathrm{dBm})\end{array}$ & $\begin{array}{c}\text { Max. pointing } \\
\text { error } \\
(\mu \mathrm{rad})\end{array}$ & $\begin{array}{c}\text { Received } \\
\text { power } \\
(\mathrm{dBm})\end{array}$ & $\begin{array}{c}\text { Max. pointing } \\
\text { error } \\
(\mu \mathrm{rad})\end{array}$ & $\begin{array}{c}\text { Received } \\
\text { power } \\
(\mathrm{dBm})\end{array}$ \\
\hline Clear air & 8.4 & -44.39 & 7.75 & -31.93 & 8.35 & -45.32 & 7.7 & -32.94 \\
\hline Haze & 8.05 & -43.55 & 7.40 & -31.64 & 8.05 & -45.48 & 7.35 & -32.69 \\
\hline Light fog & 7.40 & -43.84 & 6.65 & -31.34 & 7.35 & -44.89 & 6.6 & -32.47 \\
\hline Moderate fog & 6.95 & -43.76 & 6.1 & -31.42 & 6.9 & -45.68 & 6.05 & -32.62 \\
\hline
\end{tabular}

and PIN, which indicates that more complex noise models should be taken in consideration on dealing with APD and PIN.

\subsection{Performance Analysis for FSO Link at $850 \mathrm{~nm}$}

Performance evaluation of the proposed link at $850 \mathrm{~nm}$ with NRZ-RZ line codes and APD-PIN receivers under various weather conditions is analyzed in this section. Table 4 shows the maximum pointing error evaluated for the designed link to target a BER $<10^{-9}$. In this section, the performance of $850 \mathrm{~nm}$ link in the presence of haze is analyzed. Like the pervious section, behavior of the designed link for the chosen weather condition (haze in this section) is similar for remaining weather conditions.

In haze and APD receiver, the maximum pointing error for NRZ-APD and RZ-APD is $8.05 \mu \mathrm{rad}$. A similar maximum pointing error is observed for both NRZ-APD and RZAPD. This behavior is shared through all weather conditions providing the NRZ-APD more power efficient. For haze, 10${ }^{13}$ BER is observed for NRZ-APD and $10^{-10}$ for RZ-APD. NRZ-APD is also better in BER calculations. In haze and
PIN receiver, $7.40 \mu \mathrm{rad}$ is the maximum pointing error for NRZ-PIN and $7.35 \mu \mathrm{rad}$ for RZ-PIN. The received power levels in all weather conditions show that, NRZ-PIN is better. BER analysis yields that NRZ-PIN achieves better BER levels for all weather conditions at $850 \mathrm{~nm}$.

\subsection{Performance Analysis for the FSO Link at $1550 \mathrm{~nm}$}

Analysis done for the previous two sections is repeated in the following analysis for $1550 \mathrm{~nm}$. BER $<10^{-9}$ is targeted through maximum pointing error and received power shown in Table 5. In this section, moderate fog weather condition for $1550 \mathrm{~nm}$ is analyzed. For APD receivers in presence of moderate fog, NRZ-APD maximum pointing error is 11.85 $\mu \mathrm{rad}$ where RZ-APD maximum pointing error is $11.7 \mu \mathrm{rad}$. RZ-APD received signal is more attenuated than NRZ-APD in all weather conditions. For moderate fog, NRZ-APD and RZ-APD have BER level of $10^{-10}$.

Performance analysis for PIN shows, in the presence of moderate fog, the maximum pointing error for NRZ-PIN and RZ-PIN is $10.7 \mu \mathrm{rad}$ and $10.6 \mu \mathrm{rad}$, respectively. In all weather conditions, NRZ-PIN received signal power levels is 
Table 5. Maximum Pointing Errors and Received Signal's Power at Different Weather Conditions for NRZ-RZ Line Codes and APD-PIN receivers, $\lambda=1550 \mathrm{~nm}$

\begin{tabular}{|c|c|c|c|c|c|c|c|c|}
\hline Modulation Technique & \multicolumn{4}{|c|}{ NRZ } & \multicolumn{4}{|c|}{$\mathbf{R Z}$} \\
\hline Receiver Type & \multicolumn{2}{|c|}{ APD } & \multicolumn{2}{|c|}{ PIN } & \multicolumn{2}{|c|}{ APD } & \multicolumn{2}{|c|}{ PIN } \\
\hline & $\begin{array}{c}\text { Max. pointing } \\
\text { error } \\
(\mu \mathrm{rad})\end{array}$ & $\begin{array}{l}\text { Received } \\
\text { power } \\
(\mathrm{dBm})\end{array}$ & $\begin{array}{c}\text { Max. pointing } \\
\text { error } \\
(\mu \mathrm{rad})\end{array}$ & $\begin{array}{c}\text { Received } \\
\text { power } \\
(\mathrm{dBm})\end{array}$ & $\begin{array}{c}\text { Max. pointing } \\
\text { error } \\
(\mu \mathrm{rad})\end{array}$ & $\begin{array}{c}\text { Received } \\
\text { power } \\
(\mathrm{dBm})\end{array}$ & $\begin{array}{c}\text { Max. pointing } \\
\text { error } \\
(\mu \mathrm{rad})\end{array}$ & $\begin{array}{c}\text { Received } \\
\text { power } \\
(\mathrm{dBm})\end{array}$ \\
\hline Clear air & 14.55 & -41.12 & 13.6 & -31.58 & 14.4 & -41.50 & 13.5 & -32.54 \\
\hline Haze & 14.15 & -41.13 & 13.2 & -31.86 & 14 & -41.55 & 13.1 & -32.84 \\
\hline Light fog & 12.95 & -40.82 & 11.9 & -31.51 & 12.85 & -41.83 & 11.8 & -32.59 \\
\hline Moderate fog & 11.85 & -41.09 & 10.7 & -31.84 & 11.7 & -41.75 & 10.6 & -33 \\
\hline
\end{tabular}

better than RZ-PIN. For moderate fog, NRZ -PIN and RZPIN achieved a BER of $10^{-10}$.

\subsection{Comprehensive Study}

Performance analysis for the proposed FSO link at various wavelengths for different weather conditions is evaluated. In this section, based on the preceding analysis for selectivity of better performance of modulation techniques, operation wavelength and type of receiver are introduced. Similarities and differences between NRZ-APD and RZ-APD for different wavelengths are investigated where the pointing error allowance and received signal power and BER levels are compared.

For $785 \mathrm{~nm}$, NRZ-APD and RZ-APD show a gradual decrease in the pointing error allowance as weather conditions deteriorate more. Despite that NRZ-APD and RZ-APD have the same maximum pointing error allowance, NRZAPD is more power efficient and achieves better levels in BER than RZ-APD. Approximate performance with slight changes between NRZ-APD and RZ-APD in maximum pointing error allowance is observed for $850 \mathrm{~nm}$ system operation through different weather conditions. Considering received signal power and BER levels, NRZ-APD is better than RZ-APD. However, NRZ-APD at $850 \mathrm{~nm}$ pointing error allowance is higher than NRZ-APD at $785 \mathrm{~nm}$ in different weather conditions. The received signal power for NRZAPD at $785 \mathrm{~nm}$ is more efficient than NRZ-APD at $850 \mathrm{~nm}$ through all weather conditions.

For $1550 \mathrm{~nm}$, NRZ-APD pointing error allowance is more than RZ-APD in different weather conditions matching with the previous results for $785 \mathrm{~nm}$ and $850 \mathrm{~nm}$ FSO link operations. While detailed analysis for received signal power and BER levels shows differences. Received signal power for NRZ-APD is slightly efficient than RZ-APD, where BER levels are approximately equal. Pointing error allowance at $1550 \mathrm{~nm}$ shows a better performance for NRZ-APD than RZ-APD.

Here, similarities and differences between NRZ-PIN and RZ-PIN are presented. For $785 \mathrm{~nm}$, NRZ-PIN and RZ-PIN achieve the same pointing error allowance through different weather conditions. Received signal power and BER show a better performance for NRZ-PIN in all weather conditions.
Analysis shows the following differences between 850 $\mathrm{nm}$ and $785 \mathrm{~nm}$ operations for NRZ-PIN and RZ-PIN. Pointing error allowance for $850 \mathrm{~nm}$ shows slightly changes rather than equality in $785 \mathrm{~nm}$. BER levels for $850 \mathrm{~nm}$ are the same in clear air and haze and are better in light fog and moderate fog. Despite of $785 \mathrm{~nm}$ operations, NRZ-PIN is better than RZ-PIN in all weather conditions. Analysis for $1550 \mathrm{~nm}$, NRZ-PIN shows a notable increase in pointing error allowance compared to RZ-PIN in all weather conditions. BER levels for NRZ-PIN and RZ-PIN are the same although NRZ-PIN received signal power is more efficient.

Finally, based on the observed pointing error allowance, received signal power, BER evaluation and system specs in Section 2.3, $1550 \mathrm{~nm}$ NRZ-APD operation achieves the best performance for the proposed FSO link. Choosing $1550 \mathrm{~nm}$ as the suitable operation wavelength for the proposed FSO link shows a fair agreement with the mathematical model. This is clear by observing attenuation levels mentioned in Table 2.

APD and PIN receivers have major differences in physical structures and operation. Presented analysis for FSO link states that APD is preferable for FSO communication taking into account power levels of the transmitters, types of noise and latest FSO vendors' specs mentioned in Section 2.3. This result is concluded through monitoring pointing error allowance.

\section{CONCLUSION}

In this paper, a detailed study for FSO link based on vendors' specifications is presented. Analytical analysis and link investigation concluded that, NRZ line code with $1550 \mathrm{~nm}$ operating wavelength utilizing APD receiver in different weather conditions achieves a remarkable performance in order to keep an acceptable received signal power and BER levels.

Pointing error effect on the performance of the FSO link is a critical aspect especially in deteriorating weather conditions. Pointing error angles in the range of micro radians could lead to a link failure. Less directed beams, auto tracking systems, multi-beaming systems and multi-receiving systems could be an appropriate modification to be applied on FSO link in order to overcome pointing error effects. 
This study provides designers with a wide range of system conditions that can be applied practically and explore the idea of trade off between different configuration selections.

\section{CONFLICT OF INTEREST}

The authors confirm that this article content has no conflicts of interest.

\section{ACKNOWLEDGEMENTS}

Declared none.

\section{REFERENCES}

[1] S. Bloom, E. Korevaar, J. Schuster and H. Willebrand, "Understanding The Performance of Free-Space Optics," J Opt. Netw., vol. 2 , no. 6 , pp. $178-200$, June. 2003

[2] W. Zhang, S. Hranilovic and C. Shi, "Soft-Switching Hybrid FSO/RF Links Using Short-length Raptor codes: Design and Implementation," IEEE J. Sel. Area Commun., vol. 27, no. 9, pp. 1-11, December. 2009.

[3] S. Arnon, "Optical Wireless Communications,"In Encyclopedia of Optical Engineering, pp. 1866-1886, New York, USA, 2000.

[4] W.-D. Zhong, S. Fu and C. Lin. "Performance Comparison of Different Modulation Formats Over Free-Space Optical (FSO) Turbulence Links With Space Diversity Reception Technique," IEEE Photon. J., vol. 1, no. 6, pp. 277-285, December. 2009.

[5] S. Rajbhandari, Z. Ghassemlooy, J. Perez, H. Le Minh, M. Ijaz, E. Leitgeb, G. Kandus3 and V.Kvicera, "On The Study of The FSO Link Performance Under Controlled Turbulence and Fog Atmospheric Conditions," 11th International Conference on Telecommunications, ConTEL, Graz, Austria, pp. 223-226, June. 2011.

[6] H. Manor and S. Arnon, "Performance of an Optical Wireless Communication System as a Function of Wavelength," Appl. Opt., vol. 42, no. 21, pp. 4285-4294, July. 2003.

[7] X. Liu, "Free-Space Optics Optimization Models for Building Sway and Atmospheric Interference Using Variable Wavelength," IEEE Trans. Commun., vol. 57, no. 2, pp. 492-498, February. 2009.

[8] D. K. Borah and D. G. Voelz, "Pointing Error Effects on FreeSpace Optical Communication Links in The Presence of Atmospheric Turbulence," J. Lightwave. Technol., vol. 27, no. 18, pp. 3965-3973, September. 2009.

[9] H. G. Sandalidis, T. A. Tsiftsis, G. K. Karagiannidis and M. Uysal., "BER Performance of FSO Links over Strong Atmospheric Turbulence Channels with Pointing Errors," IEEE Commun. Lett., vol. 12, no. 1, pp. 44-46, January. 2008.

[10] J. A. Shaw, "Radiometry and the Friis Transmission Equation," Montana State University, 2005. http://www.coe.montana.edu/ee/rwolff/EE548/sring05\%20papers/F riis_Radiometric_2005Feb9.pdf

[11] I. I. Kim, B. McArthur and E. Korevaar, "Comparison of Laser Beam Propagation at $785 \mathrm{~nm}$ and $1550 \mathrm{~nm}$ in Fog and Haze for Optical Wireless Communications," In: Proc. SPIE 4214, February. 2001, pp. 26-37.

[12] M. S. Awan, Marzuki, E. Leitgeb, F. Nadeem, M. S. Khan and C. Capsoni, "Weather effects impact on the optical pulse propagation in free space," In: Proc. $69^{\text {th }}$ Vehicular Technology Conference (VTC), Barcelona, April. 2009. pp. 1-5.

[13] M. S. Awan, Marzuki, E. Leitgeb, F. Nadeem, M. S. Khan and C. Capsoni, "Fog attenuation dependence on atmospheric visibility at two wavelengths for fso link planning," Loughborough Antennas \& Propagation Conference, UK, pp. 193-196, November. 2010.

[14] W. O. Popoola and Z. Ghassemlooy, "BPSK subcarrier intensity modulated free space optical communications in atmospheric turbulence," J. Lightwave. Technol., vol. 27, no. 8, pp. 967-973, April. 2009.

[15] W.O. Popoola, Z. Ghassemlooy, C.G. Lee and A.C. Boucouvalas "Scintillation effect on intensity modulated laser communication systems - a laboratory demonstration," J. Optic Laser Technol., vol. 42, no. 4, pp. 682-692, December. 2009.

[16] K. Kiasaleh, "Performance of APD-Based, PPM free-space optical communication systems in atmospheric turbulence," IEEE Trans. Commun., vol. 53, no. 9, pp.1455-1461, September. 2005.

[17] F. Xu, M. Ali Khalighi and S. Bourennane, "Impact of Different Noise Sources on the Performance of PIN- and APD-based FSO Receivers," $11^{\text {th }}$ International Conference on Telecommunications, ConTEL, Graz, Austria, pp. 211-218, June 2011.

[18] A. A. Farid and S. Hranilovic, "Outage Capacity Optimization for Free Space Optical Links with Pointing Errors," J. Lightwave Technol., vol. 25 , no. 7, pp. 1702-1710, July 2007.

[19] M. Ijaz, Z. Ghassemlooy, S. Ansari, O. Adebanjo, H. Le Minh and S. Rajbhandari and A. Gholami, "Experimental Investigation of the Performance of Different Modulation Techniques under Controlled FSO Turbulence Channel," $5^{\text {th }}$ Inter Sym. Telecom., Tehran, Iran, pp. 59-64, December 2010.

[20] M. Uysal, J. Li and M. Yu. "Error Rate Performance Analysis of Coded Free-Space Optical Links over Gamma-Gamma Atmospheric Turbulence Channels," IEEE Trans. Wireless. Commun., vol. 5, no. 6, pp. 1229-233, June 2006.

[21] M. Al Naboulsi, H. Sizun and F. de Fornel, "Fog Attenuation Prediction for Optical and Infrared Waves," J. Opt. Eng., vol.43, no.2, pp. 319-329, February 2004.

[22] G.P. Agrawal, Fiber Optic Communication Systems, John Wiley \& Sons: New York, 2002.

[23] fSONA Optical Wireless. [Online]. Available: http://www.fsona.com

[24] Canobeam. [Online]. Availble: http://www.canobeam.com/

[25] LIGHTPOINTE Wireless. [Online]. Available: www.lightpointe.com

[26] Applied Optoelectronics. [Online]. Available: http://www.aoinc.com.

[27] HAMAMATSU. [Online]. Available: http://www.hamamatsu.com/

[28] A. Prokes, "Atmospheric Effects on Availability of Free Space Optics Systems," J. Opt. Eng., vol. 48. no. 6, pp. 1-10, June 2009.

[29] M. Ijaz, Z. Ghassemlooy, S. Ansari, O. Adebanjo, H. Le Minh and S. Rajbhandari and A. Gholami, "Experimental investigation of the performance of ook-nrz and rz modulation techniques under controlled turbulence channel in fso systems," the 11th annual post graduate symposium on the convergence of telecommunications, networking and broadcasting (PGNet 2010), Liverpool, UK, June 2010.

[30] Agrawal, G.P. and Dutta, N.K., Semiconductor Laser, $2^{\text {nd }}$ ed., Van Nostrand Reinhold: New York, N.Y., 1993.

(C) Mohammed et al.; Licensee Bentham Open.

This is an open access article licensed under the terms of the Creative Commons Attribution Non-Commercial License (http://creativecommons.org/licenses/by-nc/3.0/) which permits unrestricted, non-commercial use, distribution and reproduction in any medium, provided the work is properly cited. 